\title{
DESARROLLO DE LA COMPETENCIA COMUNICATIVA INTERCULTURAL EN UN PROGRAMA DE ADQUISICIÓN DE LA LENGUA CRIOLLO HAITIANA EN CHILE
}

\author{
Developing Intercultural Communicative Competence in an Haitian Creole \\ LANGUAGE ACQUISITION PROGRAM IN CHILE
}

DÉVELOPPEMENT DE LA COMPÉTENCE COMMUNICATIVE INTERCULTURELLE DANS UN PROGRAMME D'ACQUISITION DE LA LANGUE CRÉOLE HAÏTIENNE AU CHILI

\author{
Valeria Sumonte Rojas \\ Doctora en Educación, Universidad \\ Católica del Maule, Talca, Chile. \\ Directora del Instituto de Estudios \\ Generales y académica del Depar- \\ tamento de Idiomas, Facultad de \\ Educación, Universidad Católica del \\ Maule, Talca, Chile. \\ Avenida San Miguel, N. ${ }^{\circ} 3605$, \\ Talca, Chile. \\ vsumonte@ucm.cl \\ https://orcid. \\ org/0000-0001-8873-1892
}

Este artículo se origina como resultado de la investigación desarrollada para obtener el grado de doctor en Educación, titulada: "Diversidad lingüística e identidad cultural: el caso del criollo haitiano", en el Doctorado en Educación en Consorcio, Universidad Católica del Maule, Talca (Chile), 15 de marzo de 2019. La investigación fue patrocinada por el Proyecto FONDECYT DE INICIACIÓN 11190448 : "Diálogo entre cultura y lengua como elemento facilitador de la inclusión de migrantes adultos no hispanoparlantes que residen en las regiones del Maule y Metropolitana", financiado por la Comisión Nacional de Investigación Científica y Tecnológica de Chile (CONICYT).

\section{RESUMEN}

El objetivo del artículo es describir los resultados de un programa de adquisición de lengua criollo haitiano, cuyo objetivo era promover la competencia comunicativa intercultural de profesionales chilenos y favorecer la inclusión de las personas migrantes. El programa, basado en la teoría de la competencia comunicativa intercultural y con una duración de 50 horas, inicia con tres sesiones en las que se muestra qué significa el proceso migratorio, se da a conocer el país de origen de los migrantes por migrantes, quienes se constituyen en mediadores lingüísticos, y se exponen aspectos lingüísticos propios del español y del criollo haitiano. Para la recolección de los datos sobre los resultados del programa se usa una entrevista y una prueba de habilidades lingüísticas, dirigida a los participantes, oficiales de la Policía de Investigaciones y profesionales de la salud, las cuales se aplican al finalizar la implementación del mismo. Los resultados dan cuenta de que el programa favorece el vínculo entre individuos culturalmente diferentes, pues está basado en la interrelación de los participantes en igualdad de conocimientos, y promueve la participación de los aprendices en la construcción de su aprendizaje y la anticipación al contexto al cual se enfrentarán. Lo anterior facilita la inclusión de las personas migrantes, cuyo idioma difiere del de la sociedad de acogida, permitiendo mayor posibilidad de insertarse en ámbitos laborales, educativos y culturales.

Palabras clave: inclusión; competencia comunicativa intercultural; migración; adquisición de lenguas; criollo haitiano; Chile.

\section{Abstract}

This article aims to describe the results of a Haitian Creole language acquisition program, looking to foster intercultural communicative competence among Chilean officers, and favor migrant inclusion. The program, based on the intercultural communicative competence, had a duration of 50 hours, beginning with three sessions that present what the migration process means, the country of origin of the migrants, delivered by migrants, who then become linguistic mediators, and several linguistic aspects of Spanish and Haitian Creole languages. Data on the results of the program were collected through an interview and a language skills test, administered to the participants, to officers from the Investigation Police, and

Received: 2018-12-11 / Accepted: 2019-07-10 / Published: 2020-01-28

http://www.doi.org/10.17533/udea.ikala.v25n01a09 
to health care practitioners, at the end of its implementation. The results show that the program favors the link between individuals from different cultural backgrounds, since it relies upon the exchange of participants having equally valued knowledge, and promotes the participation of learners in managing their learning and anticipating the context they will face. This facilitates the inclusion of migrant people, speaking a different language from that of the host society, which gives them a greater likelihood to successfully insert themselves in labor, educational and cultural settings.

Keywords: inclusion; intercultural communicative competence; migration; Haitian Creole; language acquisition; Chile.

\section{RÉSUMÉ}

Cet article vise à décrire les résultats d'un programme d'acquisition de la langue créole haïtienne, dessiné pour promouvoir la compétence communicative interculturelle des fonctionnaires chiliens et favoriser l'inclusion des migrants. Le programme, basé sur la théorie de la compétence communicative interculturelle et d'une durée de 50 heures, commence avec trois sessions dans lesquelles le processus de migration, ainsi que le pays d'origine des migrants par des migrants qui deviennent médiateurs linguistiques, et des aspects linguistiques du créole espagnol et haïtien sont présentés. Pour la collecte de données sur les résultats du programme, un entretien et un test de compétences linguistiques ont été utilisés, appliqués aux participants, aux officiers de la Police d'investigations et aux professionnels de la santé, appliqués à l'issue de sa mise en œuvre. Les résultats montrent que la méthode d'enseignement et d'apprentissage favorise le lien entre des individus culturellement différents, car elle est basée sur l'interrelation des participants à connaissances égales, et favorise la participation des apprentis à la construction de leur apprentissage ainsi que leur anticipation au contexte auquel ils seront confrontés. Cela facilite l'inclusion des migrants, dont la langue differe de celle de la société d'accueil, ce qui donne une plus grande possibilité d'accéder au travail, à l'éducation et à la culture.

Mots clés : inclusion ; compétence en communication interculturelle ; migration ; acquisition des langues; langue créole haïtienne ; Chili. 


\section{Introducción}

Según António Guterres, secretario de la ONU, "El siglo XXI será el de los pueblos en movimiento" (2008, citado en Moreno, 2013, p. 80). Esto ocurre a nivel mundial, y Chile no es la excepción: con una población cercana a los 18 millones de habitantes, se ha convertido en un polo atractivo para los colectivos migratorios, donde el $6,1 \%$ de la población nacional es inmigrante (Chile, Instituto Nacional de Estadísticas [INE], 2018). En el movimiento migratorio experimentado por el país en estos últimos años (véase Tabla 1), resalta la llegada exponencial de un grupo de ciudadanos no hispanohablante,

Tabla 1 Número de migrantes haitianos en el 2002 en Chile, e ingreso, salida y permanencia en los últimos años

\begin{tabular}{cccc}
\hline Año & Ingreso & Salida & Permanencia \\
\hline 2002 & - & - & 50 \\
2013 & 1.931 & 203 & 1.728 \\
2014 & 3.844 & 257 & 3.587 \\
2015 & 12.256 & 405 & 11.851 \\
2016 & 42.000 & 1.439 & 40.561 \\
2017 & 113.125 & 4.669 & 108.456 \\
Total & $\mathbf{1 7 3 . 1 5 6}$ & $\mathbf{6 . 9 7 3}$ & $\mathbf{1 6 6 . 2 3 3}$ \\
\hline
\end{tabular}

Fuente: Policía de Investigaciones (PDI, 2018). como lo es el colectivo haitiano (Navarrete, 2015; Toledo, 2016).

En el año 2002 residían, en el país, 50 personas provenientes de Haití (Santibáñez y Maza, 2015); en 2016 ingresaron 42.000; durante el 2017, el ingreso fue de 113.125 personas, lo que corresponde a un $169 \%$ más de los que ingresaron el 2016. Por lo tanto, actualmente residen en al país 166.233 migrantes haitianos. Lo anterior, sin contar a quienes han llegado posteriormente, donde, por ejemplo, entre enero y marzo de 2018 el ingreso fue de 33.433 personas (Policía de Investigaciones -PDI-, 2018).

En relación con la migración hacia la región del Maule (una de las 16 regiones en las cuales está organizado Chile), esta corresponde a 11.474 personas, y en específico hacia la ciudad de Talca, donde se lleva a cabo esta investigación, es de 5.626 personas. Como se muestra en la Tabla 2, el $45 \%$ (725) de la migración haitiana que llega a la región permanece en la ciudad de Talca. Respecto al número de personas que hablan una lengua distinta al español, no hay datos específicos que den cuenta de esta característica.

En términos del país, este grupo migratorio está en quinto lugar de ingreso después de Perú,

Tabla 2 Migración a la región del Maule y a la ciudad de Talca, por país/continente y género

\begin{tabular}{lcccccc}
\hline \multirow{2}{*}{ País/Continente } & \multicolumn{3}{c}{ Región del Maule } & \multicolumn{3}{c}{ Ciudad de Talca } \\
\cline { 2 - 7 } & Hombre & Mujer & Total & Hombre & Mujer & Total \\
\hline África & 33 & 12 & 45 & 17 & 2 & 19 \\
Asia & 213 & 137 & 350 & 88 & 63 & 151 \\
Caribe & 180 & 185 & 365 & 125 & 122 & 247 \\
Centroamérica & 113 & 131 & 244 & 59 & 75 & 134 \\
Europa & 675 & 585 & 1.260 & 350 & 281 & 631 \\
Haití & 1.207 & 397 & 1.604 & 549 & 176 & $\mathbf{7 2 5}$ \\
Norteamérica & 193 & 153 & 346 & 97 & 68 & 165 \\
Oceanía & 19 & 11 & 30 & 10 & 6 & 16 \\
País no declarado & 70 & 55 & 125 & 36 & 16 & 52 \\
Sudamérica & 3.464 & 3.641 & 7.105 & 1.697 & 1.789 & 3.486 \\
Total & $\mathbf{6 . 1 6 7}$ & $\mathbf{5 . 3 0 7}$ & 11.474 & $\mathbf{3 . 0 2 8}$ & $\mathbf{2 . 5 9 8}$ & $\mathbf{5 . 6 2 6}$ \\
\hline
\end{tabular}

Fuente: Basado en información del Instituto Nacional de Estadísticas (Chile, INE, 2018) 
Colombia, Venezuela y Bolivia, pero se ha hecho visible, debido a su color de piel oscuro y a que, en su mayoría, hablan un idioma diferente al español: el criollo haitiano. Dado que los chilenos no tienen dominio del criollo haitiano, se dificulta el entendimiento entre el colectivo migratorio y la sociedad de acogida.

Adicionalmente, la Tabla 3 muestra el nivel de escolaridad del grupo migratorio haitiano, de acuerdo con lo declarado por ellos en el Censo 2017 (Chile, INE, 2018). Allí se observa que una minoría de personas provenientes de Haití son profesionales y un tercio de haitianos cuenta solo con estudios de enseñanza básica, lo que hace más compleja su inserción a nivel laboral. Por consiguiente, y con el estigma de provenir de un país vulnerable (Toledo, 2016), se hace urgente adoptar estrategias

Tabla 3 Escolaridad migrantes haitianos residentes en Talca, por edad de grandes grupos

\begin{tabular}{lcccc}
\hline \multicolumn{1}{c}{$\begin{array}{c}\text { Curso más } \\
\text { alto }\end{array}$} & \multicolumn{3}{c}{ Edad } & Total \\
\cline { 2 - 4 } & $\mathbf{0 - 1 4}$ & $\mathbf{1 5 - 6 4}$ & $\mathbf{6 5}$ o más & \\
\hline Prebásica & 10 & 1 & - & 11 \\
Educación básica & 3 & 485 & 4 & 492 \\
Humanista & - & 496 & - & 496 \\
Técnico & - & 260 & 2 & 262 \\
profesional & & & & \\
Profesional & - & 166 & - & 166 \\
Posgrado & - & 2 & 1 & 3 \\
Total & $\mathbf{1 3}$ & 1.410 & 7 & 1.430 \\
\hline
\end{tabular}

Fuente: Instituto Nacional de Estadísticas (Chile, INE, 2018)

que faciliten la integración de este colectivo a la sociedad de acogida.

De acuerdo con lo expresado por investigaciones realizadas en el país acerca de este grupo migratorio (Navarrete, 2015; Rojas Pedemonte, Silva, Amode, Vásquezy Orrego, 2016; Rojas Pedemonte y Koechlin, 2017; Toledo, 2016), uno de los requerimientos más urgentes corresponde a la capacidad de entendimiento intercultural mediante la lengua.
En el contexto del movimiento de la población a nivel mundial del siglo XXI, son tanto los recién llegados como la población de acogida los responsables de comprenderse y reconocerse mutuamente, y de transitar hacia la integración.

En general, en la realidad actual, el énfasis está puesto en la manera en que el inmigrante debe rápidamente aprender la lengua del país de acogida, para insertarse en la sociedad (Arroyo, 2012; Huguet, 2008, 2014; Oller y Vila, 2008). Una de las condicionantes que promueve la integración social y económica de los inmigrantes se basa principalmente en las competencias lingüísticas del idioma de acogida que logren adquirir (Chiswick y Miller, 2001; Sancho, 2013; Toledo, 2016).

Sin embargo, las propuestas dirigidas al dominio del idioma de la comunidad que migra por parte de la sociedad de acogida son casi inexistentes. Las que se pueden encontrar, están relacionadas con aquellas lenguas que cuentan con un gran número de hablantes, como lo son el español y el chino mandarín. Por ejemplo, en Estados Unidos se han establecido programas de español, por la alta migración de ciudadanos cubanos (Ramos, 2006). En el país, un programa de enseñanza y aprendizaje del español que se destaca es el desarrollado por la Universidad de Chile, dirigido a personas provenientes de diferentes países (Universidad de Chile, 2016).

Por consiguiente, el objetivo de este trabajo es describir el "Programa de adquisición de la lengua criollo haitiano" y sus resultados, un programa educativo de enseñanza y aprendizaje de esta lengua, que promueve la competencia comunicativa intercultural y favorece la inclusión de las personas migrantes. El programa, implementado en Talca, se desarrolla a partir de la interrelación horizontal entre los miembros de la comunidad de acogida y la migratoria, impulsando estrategias que integren la lengua y la cultura de ambos grupos. De la misma forma, se incentiva a los inmigrantes haitianos a transformarse en referentes lingüísticos y culturales, con el fin de favorecer la adquisición de 
la lengua y lo que esta trae consigo, a partir de nativos lingüísticos y culturales (Sumonte, Sanhueza, Friz y Morales, 2018).

\section{El programa de adquisición de la lengua criollo haitiano}

Este programa busca promover las conclusiones de un informe sobre la multiplicidad de lenguas en Europa, que dice:

\begin{abstract}
Del mismo modo que se animaría a los inmigrantes a que adoptaran plenamente la lengua del país de acogida y la cultura que transmite, sería justo y útil que las lenguas identitarias de los inmigrantes formaran también parte del grupo de lenguas cuyo aprendizaje se aconseja a los europeos (Maalouf, 2008, p. 22).
\end{abstract}

Así mismo, se desarrollan las actividades dentro del aula, intencionando el entendimiento intercultural entre dos comunidades que se han encontrado de manera repentina y que no tienen mayor información una de la otra. Para ser un hablante que respete los distintos referentes culturales, se debe no solo comunicar información, sino también desarrollar relaciones humanas con personas de otras lenguas y culturas (Byram, Gribkova y Starkey, 2002).

$\mathrm{Al}$ referirnos a la cultura, esta se comprende como los valores, estilos de vida y representaciones simbólicas (cosmovisiones) que las personas utilizan al relacionarse con otras y en su entendimiento acerca del mundo (Rey von Allmen, 2010). De esta manera, el programa es conducido hacia la interculturalidad, cuya finalidad es construir el encuentro entre sujetos que comparten un determinado espacio, pero que pertenecen a sociedades y culturas diferentes.

\section{El programa adopta la teoría de la competencia} comunicativa intercultural, entendida dicha competencia como las habilidades cognitivas, afectivas y actitudinales que emplean los individuos culturalmente diferentes para favorecer un grado de comunicación eficaz, estableciendo comportamientos apropiados y efectivos en determinados contextos sociales y culturales (Chen y Starosta, 1998). "El concepto de competencia comunicativa intercultural intenta responder a la necesidad de ofrecer un modelo para la integración de lengua y cultura en la enseñanza de lenguas” (Areizaga, 2001, p. 162).

Asimismo, el programa sustenta su implementación bajo tres principios:

1. Hipótesis de interdependencia lingüistica (Cummins, 1979, 1981). Implica la valoración y el (re)conocimiento de la lengua materna (español chileno), pero, al mismo tiempo, el conocimiento de la lengua en aprendizaje (criollo haitiano) y, por consiguiente, la cultura, sin perder o rechazar la de origen.

2. Aprendizaje situado y colaborativo. En el aprendizaje situado, el aprender y el hacer son conductas inseparables (Díaz, 2003). Así, la adquisición de la lengua se dará en la medida en que lo trabajado en el contexto de aula corresponda a las necesidades e intereses de los aprendices, y que les permita poner en práctica lo aprendido al término del encuentro educativo. Como lo mencionan Lave y Wenger (1991), se aprende en la experiencia, donde los aprendices aplican lo aprendido en situaciones relevantes de la vida real.

El aprendizaje colaborativo (Díaz, 2003), por su parte, considera la interacción en un plano de igualdad de los participantes en cada encuentro. Estos, de forma colaborativa, construyen conocimiento intercultural, ya que cada uno de ellos no solo es responsable de su propio aprendizaje, sino que también es parte del aprendizaje del otro.

3. Los mediadores lingüisticos (ML). Son migrantes haitianos, seleccionados a partir de cursos de español que se dictaban en la ciudad de Talca y donde ellos participaban. En dichos cursos se visualizan aquellos aprendices haitianos que tienen dominio del español y están interesados en transformarse en ML.

Su relevancia radica en el proceso de andamiaje en pos del aprendizaje de los participantes del 
encuentro educativo (Antón, 2010; León, 2014), la negociación en conjunto de los significados y la (co)construcción de los saberes. Los participantes contribuyen a esta generación de conocimiento y de interacción de saberes, lo que implica un aprendizaje colaborativo y de horizontalidad, donde el ML es el guía de cada encuentro; pero, al mismo tiempo, se posiciona al nivel de los demás (aprendices), en el momento en que aprende de los saberes compartidos por los otros miembros del grupo y aporta desde su propia visión de mundo.

De esta manera, se cumple parcialmente lo que establece Serra y Vila (2005): los ML tienen el dominio de ambas lenguas, pero no cuentan con competencias profesionales pedagógicas, de acuerdo con lo que entendemos en Chile por estas competencias, que implica, entre otras cosas, haber sido entrenado para enseñar, por un periodo entre cuatro a cinco años, en una institución de nivel superior. Por lo tanto, antes de iniciar el programa, se acuerdan horarios semanales de trabajo, los que se realizan los días sábados por la mañana. En estas sesiones, los ML se preparan didácticamente en la forma de compartir sus conocimientos con los aprendices.

\section{Las conversaciones iniciales}

El programa de adquisición de la lengua criollo haitiana dura 50 horas, con dos encuentros semanales, de dos horas cada uno. Para su implementación, se establecieron conversaciones iniciales entre los aprendices y los que dirigen la enseñanza $(\mathrm{ML})$, donde se acordó que el objetivo era aprender a comunicarse en el idioma criollo haitiano a un nivel elemental, que permitiera una comprensión lingüística de las necesidades de los migrantes por parte de los oficiales de la PDI y de los profesionales de la salud. Se propone un dominio del criollo haitiano a nivel A1. Según el Marco común europeo de referencia para las lenguas: aprendizaje, enseñanza, evaluación, para el logro del nivel A1, el aprendiz:

Es capaz de comprender y utilizar expresiones cotidianas de uso muy frecuente, así como, [sic] frases sencillas destinadas a satisfacer necesidades de tipo inmediato.
Puede presentarse a sí mismo y a otros, pedir y dar información personal básica sobre su domicilio, sus pertenencias y las personas que conoce.

Puede relacionarse de forma elemental siempre que su interlocutor hable despacio y con claridad y esté dispuesto a cooperar (Instituto Cervantes, 2002, p. 26).

Amedidaquetranscurríanlasconversacionesiniciales, comenzaron a surgir otros desconocimientos, como qué significaba un proceso migratorio, por qué los comportamientos de los migrantes haitianos diferían de los de la sociedad de acogida, y la dificultad para entender lo que decían, debido a su pronunciación. El resultado de estas conversaciones permite dar cuenta de lo establecido por Ferrada y Flecha (2008), quienes señalan que el conocimiento se construye y valida con todos los miembros de la comunidad educativa, lo que sienta la base para el logro de los objetivos propuestos. Por consiguiente y para responder a las necesidades identificadas, se acuerda iniciar el programa con tres diferentes charlas en tres sesiones.

La primera sesión es acerca de lo que significa el proceso migratorio. Estuvo a cargo de un miembro de la Organización Internacional para las Migraciones.

En la segunda sesión, uno de los ML relata la historia de Haití. Este explica y grafica, por medio de imágenes y desde su cosmovisión de mundo, lo que es para ellos su país de origen, el significado del periodo de esclavitud, posterior liberación e independencia, como también el origen de su cultura y su lengua. Esta sesión es un momento crucial, dado que los aprendices inician el proceso de construcción del otro a partir de los mismos referentes nativos. Como lo plantea Vilà (2009), lo que se pretende es preparar a todos, en conjunto, para compartir y aprender a comunicarnos con una sociedad plural, sin que ello implique renunciar a los propios referentes.

Lo anterior da cuenta de que el programa focaliza su accionar hacia un encuentro entre dos culturas, donde el propósito de enseñar una lengua es el medio para un encuentro intercultural. De acuerdo con Paricio, "esta apertura no solo posibilitaría la adquisición de nuevos conocimientos 
culturales, sino que facilitaría también el desarrollo de actitudes de respeto, tolerancia y comprensión del 'otro' en su diferencia' (2014, p. 218).

En la tercera sesión se muestran los diferentes órganos fonoarticulatorios, que cumplen un rol en la producción de los sonidos, ejemplificando algunas diferencias entre la producción oral del español y del criollo haitiano, lo que podría dificultar el aprendizaje.

El desarrollo de las otras sesiones del programa educativo es explicitada en el siguiente apartado.

\section{Etapas de cada sesión}

En este apartado se describe cómo se trabaja en cada una de las siguientes sesiones, lo que implica una didáctica que permite la implementación de diferentes actividades para el logro de los objetivos planteados para cada uno de los encuentros y, de esta manera, alcanzar el nivel del idioma propuesto al inicio del programa (A1). La Figura 1 muestra las etapas en las cuales se desarrolla cada clase.

Cada una de las siguientes sesiones o clases se divide en tres etapas. En la primera, con una duración de 10 minutos aproximadamente, se trabaja con los aprendices, en un solo grupo, en actividades lúdicas que están relacionadas con recordar los saberes adquiridos durante la sesión anterior; esto permite reforzar o clarificar conocimientos y saberes ya (co) construidos y evidenciar aquellos que aún no han sido internalizados. Esto se realiza mediante un proceso de metacognición, donde se hacen preguntas, se dan ideas y se entregan claves que permiten que los aprendices reconozcan el significado del léxico trabajado, estructuren frases y oraciones simples, entre otras actividades motivacionales.
En la segunda etapa, con una duración de 45 minutos, el mediador presenta los nuevos contenidos lingüísticos. Cuando los aprendices no logran comprender el conocimiento entregado, en este contexto se propicia el apoyo de un aprendiz mediador (compañero de clase) que hubiese comprendido el acto comunicativo, y usando su lengua materna (L1), que medie entre el aprendiz, el ML y los materiales didácticos, hasta que el primerologra el aprendizaje. Es decir, en el aula se promueve el uso de la L1 del aprendiz, en el entendido que facilitará el aprendizaje. Littlewood y Yu (2011) establecen que el cambio de código por parte de los mediadores no tiene influencias negativas en contextos donde la sala de clase es la principal o única fuente de exposición al idioma por parte de los aprendices, y su uso se realiza en situaciones donde el lenguaje meta no es la lengua mayoritaria.

En la tercera y última etapa, que se lleva a cabo durante una hora, los cuatro ML y los aprendices realizan la práctica de los aspectos lingüísticos trabajados durante la segunda etapa. Por ejemplo, en el contexto situado del área de salud, simulan estar en un centro asistencial, donde el ML está en el rol de enfermo y es quien requiere de atención médica, $y$ es atendido por un especialista de la salud, en este caso el aprendiz. El especialista en salud (el apren$\mathrm{diz}$ ) le formula preguntas al enfermo $(\mathrm{ML})$, usando la lengua meta, que le permita entender el estado del paciente, como: Poukisa ou isit la? (¿Por qué estás aquí?), Kisa ki ap fé w mal? (¿Qué te duele?), Kikote ou rete?(¿Dónde vives?), Depikilè ou santi w malad konsa? (¿Desde cuándo te sientes enfermo?) y Ki laj ou? (¿Qué edad tienes?) (traducido por el autor). En otras ocasiones, hay cambio de roles y es el aprendiz quien se posiciona como el paciente.

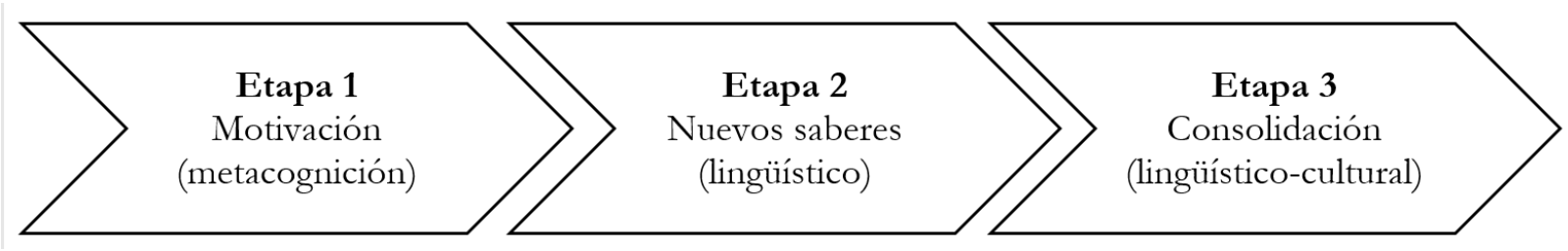

Figura 1 Etapas de cada sesión

Fuente: elaboración propia 
En contexto de la PDI, la metodología de trabajo es la misma; la diferencia radica en el vocabulario a ser utilizado y los roles desarrollados (policía y migrante). En este contexto surgen preguntas como: Kote paspó ou? (¿Dónde está tu pasaporte?), Ki kote ou travay? (¿Dónde trabajas?), Kote dokiman ou yo? (¿Dónde están tus documentos?).

En la formulación de las preguntas y respuestas, el aprendiz, en algunos casos, usa estructuras monosilábicas, pero que permiten evidenciar que existe comprensión de la pregunta, como también la capacidad lingüística de emitir una respuesta adecuada, aunque incompleta y en ocasiones gramaticalmente incorrecta, pero comprensible. Estas actividades posibilitan la interrelación entre el ML y el aprendiz, donde ambos se toman un tiempo para compartir conocimientos propios de su país de origen.

En la segunda y la tercera etapa se forman grupos, cada uno de ellos guiado por un ML. Existe una diferencia entre estas: en la segunda, los grupos estudian el mismo contenido, usando idénticos materiales didácticos y se mantiene el mismo $\mathrm{ML}$ en el grupo. En cambio, en la tercera etapa, cada ML trabaja el mismo contenido, pero a través de una actividad diferente; por lo tanto, hay cuatro mediadores, lo que implica cuatro actividades distintas, implementadas en cada grupo por 15 minutos. Terminada la actividad con el grupo (15 minutos), el mediador se mueve al siguiente por otros 15 minutos, y luego al siguiente. Es decir, rota durante la práctica por los cuatro grupos, configurando un aula en permanente dinamismo (Antón, 2010; Ferrada y Flecha, 2008).

Este modelo pedagógico favorece la diversificación de estrategias de aprendizaje, lo que posibilita la diversidad de estilos del mismo y el reconocimiento de la heterogeneidad de los participantes, en cuanto a cultura, género, rendimiento, afinidades e intereses.

De igual modo, los aprendices integran estrategias lingüísticas y culturales que les facilita desenvolverse y entender situaciones del diario vivir de la comunidad migratoria y, por su parte, los ML logran la comprensión de aspectos de la lengua y la cultura de sus aprendices, relativos a sus creencias religiosas, valores, formas de comportarse, entre otras.

Las actividades son apoyadas con materiales didácticos diseñados para esa actividad: tarjetas, guías con dibujos y grafía, juegos como el loto y lotería, rompecabezas, sopa de letras, entre otras. Así, para cada actividad y para cada contenido, se usa un material concreto y real, que permite poner el aprendizaje en un contexto situado y colaborativo.

En consecuencia, el aula y la estructura de grupo se convierten en un ambiente que favorece el establecimiento de redes de conocimiento y de interacciones colaborativas y sociales, enfatizadas por Vigotsky (1993) como el vehículo que permite el desarrollo del ser humano, lo que conduce a significados que influenciarán la construcción del propio conocimiento, lo que denomina "mediación cultural".

A continuación se presenta el estudio que pretende mostrar los resultados del programa.

\section{Método}

El presente estudio adoptó una metodología de investigación cualitativa, con un componente cuantitativo (Bisquerra, 2009; Bohr, 1958). De acuerdo con Bohr (1958), existen ciertos fenómenos que se dan a partir de una sola problematización, que no son posibles de ser observados desde una sola metodología en específico, sino que es necesario utilizar diferentes formas de entenderlos. De esta manera, el enfoque cualitativo es abordado por medio de una entrevista semiestructurada, y el cuantitativo, por una prueba de habilidades lingüísticas del criollo haitiano.

\section{Participantes}

Los participantes del estudio son oficiales de la PDI de Chile $(N=31)$ y profesionales que trabajan en seis centros de salud familiar de Talca $(N=29)$. La muestra es por accesibilidad (Bisquerra, 2009), debido a que no se tuvo participación en la selección, sino que fue realizada por la unidad de 
Recursos Humanos de ambas instituciones, focalizada en los profesionales que están en primera línea de contacto con las personas que necesitan de apoyo de ambas instituciones gubernamentales. En cuanto al género, 51,6\% son mujeres y 48,4\% son hombres, lo que corresponde a una muestra homogénea. Sus edades van desde los 28 hasta los 60 años. Todos ellos son aprendices del "Programa de adquisición de la lengua criollo haitiana”, desarrollado en Talca.

\section{Recolección de datos}

Las técnicas seleccionadas para la recolección de datos sobre el programa fueron la entrevista semiestructurada y una prueba de habilidades lingüísticas del criollo haitiano.

La entrevista semiestructurada, según Cunliffe (2010), facilita la recolección de información y sus interpretaciones, debido a que los datos se recogen a partir de las personas insertas en el mismo contexto.

Establecidos el objetivo de investigación y el desarrollo del programa, se diseñó el protocolo de entrevista, cuyas preguntas estaban enfocadas a conocer la opinión de los participantes acerca del desarrollo del programa, en el que se consideraron la metodología, los mediadores, los materiales, entre otros elementos del programa. Este protocolo fue sometido a validez de contenido, llevada a cabo por el juicio de cuatro expertos, quienes hacen parte, dos de ellos, del área de la adquisición de una segunda lengua; el tercero es un profesor de aula y el último es un especialista en métodos de investigación. Estos debían valorar los aspectos lingüísticos, de contenido, de estructura y pertinencia de la entrevista.

Tras obtener la última versión corregida, la entrevista se aplica a los profesionales de acuerdo con su disponibilidad, resguardando las condiciones de confianza, seguridad y empatía. Esta entrevista fue realizada durante los 15 días posteriores al término del programa, en el lugar de trabajo y en horario establecido por los entrevistados.
Luego, las respuestas fueron transcritas e ingresadas al programa QSR Nvivo 10, para organizar la información y realizar el análisis. A través del software, se identificaron y codificaron segmentos del texto presentes en el discurso oral de los participantes (Rodríguez, Gil y García 1999). La identificación de las entrevistas se estableció de manera aleatoria, asignando la convención: E (entrevistado) y 1-6 (según el número correspondiente a la entrevista).

Por su parte, la prueba medía el nivel de avance de las cuatro habilidades lingüísticas del criollo haitiano: dos comprensivas (comprensión auditiva y por escrito) y dos expresivas (expresión escrita y oral).

La prueba fue diseñada para ser aplicada a este grupo de aprendices, con base en evaluaciones estandarizadas desarrolladas a nivel internacional, usadas originalmente para medir el nivel del idioma inglés, y fue respondida por los aprendices el último día de clases. Lo anterior corresponde a un diseño cuasiexperimental de una sola prueba, con el grupo que desarrolla el programa, y luego se evalúa el resultado (Hernández, Fernández y Baptista, 2014). Estos autores señalan que este diseño tiene la desventaja de no permitir la comparación con una evaluación previa, y como es de grupo único, la comparación con otro grupo. Pero también afirman que solo la aplicación de una prueba final hace suponer que la muestra no conoce el contenido a trabajar.

En esta investigación, no era relevante la aplicación de la prueba inicial, debido a que a través de la encuesta demográfica que se realizó al inicio de programa a los participantes, todos ellos declaran nulo conocimiento del idioma criollo haitiano; es más, para todos, tanto el idioma como la cultura de este pueblo les era desconocida.

Luego de diseñada, se estudió la validez de contenido de la prueba, a partir de la evaluación de siete expertos: dos en el ámbito de la adquisición de una segunda lengua, académicos de una universidad en el país; un ciudadano haitiano, que cursa doctorado en salud mental en una universidad regional 
en Chile; un ciudadano haitiano, técnico en equipos médicos y traductor; un ciudadano haitiano con estudios en turismo y quien domina cuatro lenguas: inglés, francés, castellano y criollo haitiano; un experto en lingüística y con trabajos de investigación en migración haitiana, y un metodólogo. Todos ellos debieron valorar cuantitativamente cada uno de los ítems de la prueba, utilizando el siguiente criterio de relevancia: $0=$ nada relevante $(\mathrm{NR}), 1=$ poco relevante $(\mathrm{PR})$, $2=$ relevante $(\mathrm{R})$. También se proporcionó espacio en la prueba para que los jueces dejaran sus comentarios.

Los resultados de los análisis arrojaron el índice de validez de la prueba, que se muestra en la Tabla 4, el cual es considerado aceptable, según el procedimiento descrito por Tristán (2008), donde se establece 0,58 como índice mínimo de aceptación.

De acuerdo con la Tabla 4, el ítem correspondiente a la habilidad de expresión escrita no logra el mínimo de rango para ser considerado como aceptado $(0,58)$. A pesar de ello, se decide dejar este ítem, debido a que turación de una oración, donde lo más importante, conforme a los énfasis en el programa y el interés por parte de los participantes, era conocer la aplicación de los verbos, pero no sin antes modificar dicho ítem según las recomendaciones realizadas por los expertos consultados. Los datos recogidos son ingresados al software IBM SPSS versión 18.

La prueba que mide el nivel de las habilidades lingüísticas adquiridas por los aprendices es obligatoria, debido a que el programa constituye un perfeccionamiento para los profesionales de la PDI y de la salud,

Tabla 4 Índice de validez de contenido de la prueba de criollo haitiano

\begin{tabular}{llcccc}
\hline \multicolumn{2}{c}{ Habilidad } & R & PR & NR & IVC \\
\hline Comprensión & Auditiva & 6 & 1 & 0 & 0,71 \\
& Escrita & 6 & 1 & 0 & 0,71 \\
\multirow{3}{*}{ Expresión } & Escrita & 5 & 2 & 0 & 0,43 \\
& Oral & 6 & 1 & 0 & 0,71 \\
\hline
\end{tabular}

R: Relevante; PR: Poco relevante; NR: Nada relevante; IVC: Índice de validez de contenido

Fuente: Elaboración propia

y dependiendo de la nota final que obtengan, les permite subir de jerarquía y, por lo tanto, aumentar sus salarios.

Considerando lo establecido a nivel nacional en lo relativo a lo que significan las notas finales obtenidas en las pruebas, y por la importancia para los aprendices de contar con una cuantificación de sus avances en el nivel lingüístico del idioma, se estableció que las notas van de 1,0 (lo que significa que el aprendiz no logró responder de manera correcta ninguna de las preguntas realizadas) a 7,0 (nota máxima, donde todas sus preguntas fueron respondidas de manera correcta). La nota que va de $1,0 \mathrm{a}$ 3,9 corresponde a una nota insuficiente, donde no hay posibilidad de comunicarse en el otro idioma; a partir de 4,0 a 5,9, se establece que es posible comunicarse con errores y oraciones poco estructuradas, pero se logra la comprensión (nivel intermedio); a partir de 6,0 (nivel avanzado), el aprendiz ha logrado el nivel esperado, el cual es A1.

El protocolo de aplicación de la prueba implicó que la evaluación de las habilidades de comprensión auditiva y escrita, y expresión escrita, se realizara en un solo momento y con todo el grupo al mismo tiempo, con la diferencia que, para la primera (comprensión auditiva), es uno de los ML quien emite oralmente los reactivos para producir la comprensión auditiva por parte de los aprendices; esto se desarrolla durante 20 minutos. Para la segunda y la tercera habilidad (comprensión y expresión escrita), cada uno de los participantes responde en su puesto de trabajo, de manera escrita, individual y silenciosa, cada uno de los ejercicios entregados en una hoja de papel, para lo cual cuentan con 30 minutos. La expresión oral es realizada en parejas y es aplicada por uno de los ML. Para ello, se organizan cuatro esquinas - una por cada $\mathrm{ML}$ - , con una mesa y tres sillas, y en cada esquina se ubica un ML con un sobre y en ellos sus reactivos, y evalúa en los aprendices sus avances en la expresión oral.

\section{Resultados}

En este apartado se presentan los resultados que dan cuenta, en primer lugar, de los discursos de los 
aprendices sobre la implementación del programa educativo, y luego se muestra el avance en el nivel de las habilidades lingüísticas del criollo haitiano. En la Figura 2 se hace un resumen de los discursos de los participantes acerca de cada una de las actividades introductorias, que permitieron conocer el contexto al cual se enfrentarían los aprendices.

Las preguntas iniciales hechas por E3 develan una preocupación por el proceso migratorio, donde entienden esto último como una amenaza. Esta amenaza está relacionada con las percepciones negativas de la migración, que corresponden a problemáticas sociopolíticas ocasionadas por la creencia de que los migrantes son culpables de las debacles que acontecen en las sociedades de llegada, como la delincuencia, la aparición de enfermedades y la reducción de los empleos, debido a que tomarían aquellos que debiesen ser realizados por los miembros de la sociedad de acogida.

Pese a esto, en el transcurso de las sesiones en las cuales se desarrolla un intercambio de saberes entre la sociedad de acogida y los ML nativos, los discursos y la comprensión del otro - plasmados bajo la cultura de Haití (Figura 2) - , se van transformando en entender a esos otros desde otras perspectivas, lo que queda claramente establecido en el discurso de E5 y E2.

En relación con las competencias lingüísticas, en el momento en el cual se anticipa las posibles dificultades que pudiese enfrentar el aprendiz, se produce una aceptación de ellas, facilitando la producción oral.

Luego, al analizar la prueba de criollo haitiano después de aplicada, los resultados muestran avance en las habilidades lingüísticas de los aprendices, como se evidencia en la Tabla 5, lo que también queda de manifiesto en el siguiente testimonio:

Siento que con lo que aprendimos, sí podemos entablar una conversación como con palabras aisladas, pero igual entendemos, igual podemos escribir nuestras partes del cuerpo, o preguntarle si algo le duele, de dónde viene, cuántos años lleva en Chile, etc. Porque antes del programa, no podíamos hacer nada (E2).

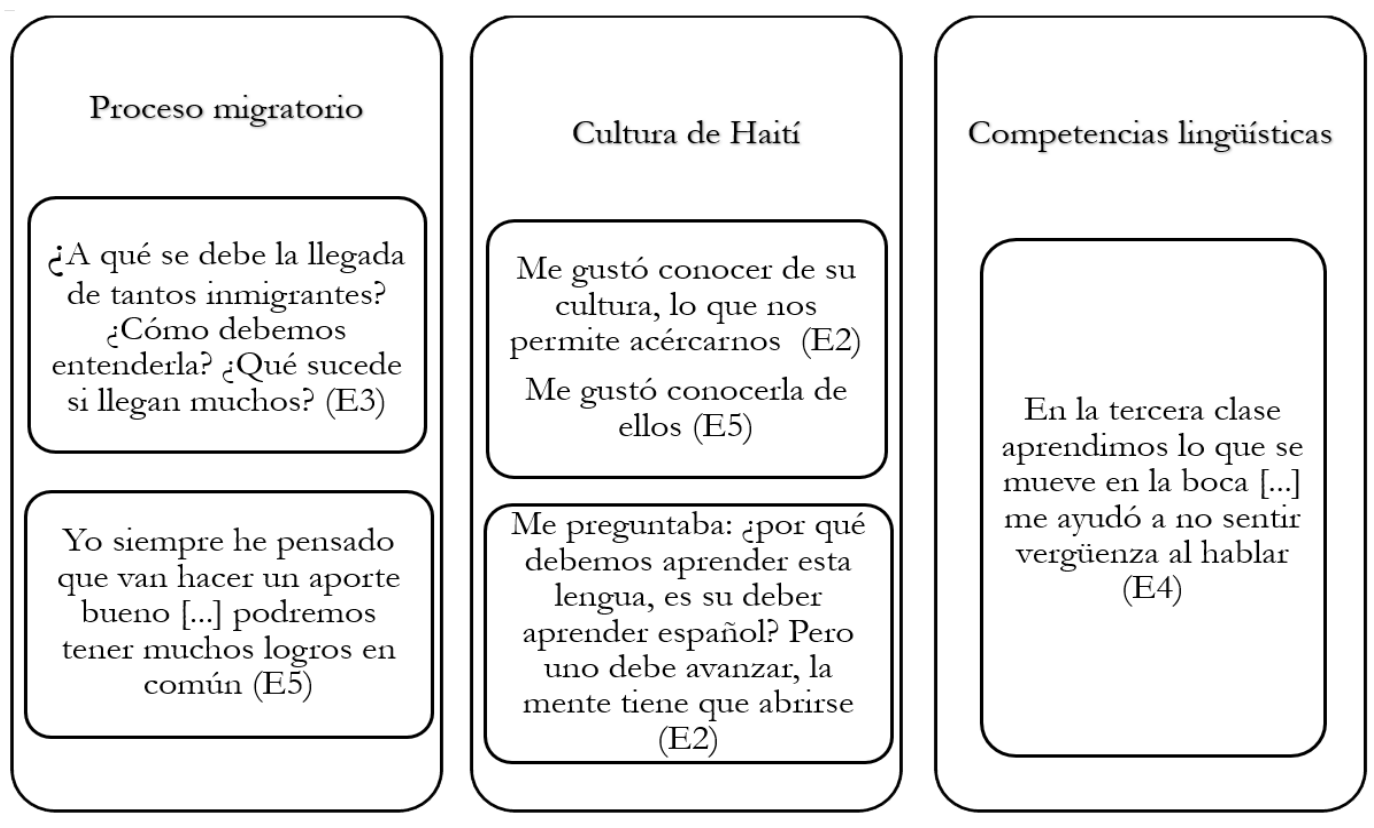

Figura 2 Discursos de los participantes

Fuente: Elaboración propia, a partir de los discursos de los entrevistados 
La Tabla 5 muestra que ningún aprendiz obtuvo la nota mínima; es decir, el 100 \% logra, en diferentes niveles, comunicarse en la lengua criollo haitiana.

Es más, el 63,4\% de los aprendices logra obtener una evaluación que indica que su nivel en el idioma es avanzado, en relación con lo establecido

Tabla 5 Rango notas finales, frecuencia y porcentaje de la prueba criollo haitiano

\begin{tabular}{lll}
\hline Rango de notas & Frecuencia & $\begin{array}{l}\text { Porcentaje } \\
(\%)\end{array}$ \\
\hline $1,0-3,9$ & 0 & 0 \\
\hline $4,0-5,9$ & 22 & 36,6 \\
\hline $6,0-7,0$ & 38 & 63,4 \\
\hline
\end{tabular}

Fuente: elaboración propia a partir de los resultados de la prueba de criollo haitiano

con el Marco común europeo de referencia para las lenguas: aprendizaje, enseñanza, evaluación (Instituto Cervantes, 2002).

Uno de los entrevistados manifiesta su sorpresa en los logros alcanzados y lo que evidenció en el momento de la evaluación:

Pensé que no iba a saber la respuesta, y entonces respondía, y estaba bien, sin pensar que estaba bien lo que yo iba a responder, era como por inercia. Entonces, yo a veces me pregunto, con respecto a las pruebas, si me fue bien o me fue mal; peroyo digo: "no sé"; pero cuando me preguntaban y yo respondía, estaba bien; entonces yo creo que algo debo haber aprendido de todo esto (E1).

En relación con la comparación en el nivel de logro de las habilidades comprensivas y expresivas, los resultados se muestran en la Tabla 6.

La Tabla 6 muestra que el mismo número de aprendices en ambas habilidades logra una calificación por sobre el 4,0. La diferencia se encuentra en el nivel de logro en las habilidades expresivas, que comprenden la habilidad de expresarse verbalmente y por escrito.

En relación con las habilidades orales, los elementos que complejizaron a los aprendices fue el producir los sonidos nasales que existen en criollo haitiano.
Tabla 6 Nivel de habilidades comprensivas y expresivas. Frecuencia y promedio

\begin{tabular}{lcccc}
\hline Niveles de & \multicolumn{4}{c}{ Habilidades } \\
\cline { 2 - 5 } logro & \multicolumn{2}{c}{ Comprensivas } & \multicolumn{2}{c}{ Expresivas } \\
& Frecuencia & Nota & Frecuencia & Nota \\
& & promedio & & promedio \\
\hline Intermedio & 22 & 5,44 & 22 & 4,7 \\
Superior & 38 & $\mathbf{6 , 4 6}$ & 38 & $\mathbf{6 , 5}$ \\
\hline
\end{tabular}

Fuente: Elaboración propia, a partir de los resultados de la prueba de criollo haitiano

En español, estos sonidos son tres: $/ \mathrm{m} /, / \mathrm{n} / \mathrm{y} / \tilde{\mathrm{n}} /$; en cambio, la lengua criollo haitiana cuenta con vocales nasalizadas, que no tenemos en español, y las mismas vocales de manera no nasalizadas. Por lo tanto, si la producción cambia en el proceso de articulación, la palabra cambia y, por ende, su significado. Lo mismo sucede de manera escrita.

Importante fue en este punto hacer una introducción al aparato fonoarticulatorio y establecer algunas diferencias entre la lengua meta y la lengua materna. Los aprendices señalan que:

\section{Fue importante, el primer día que empezamos, el cono- cer lo que se mueve dentro de la boca para poder decir cualquier sonido, y eso también me ayudó a no sentir vergüenza después a hacer los sonidos con la nariz, porque, como usted dijo, nosotros no hacemos ningún esfuerzo para hablar (E4).}

Al mismo tiempo, la dificultad anterior les hacía más complejo comprender auditivamente:

Hay que agudizar el oído en la pronunciación de las letras que traen tildes y las que son nasales; esas son las que cuestan (E5).

\section{Conclusiones}

En general, se establece que son los inmigrantes los que deben aprender la lengua del país al cual llegan, debido a que son ellos los que necesitan insertarse, como también se les facilitaría el proceso de aprendizaje, debido a su inserción en el contexto donde se habla la lengua, de acuerdo con el origen cultural inherente a esa sociedad. 
Sin embargo, el creciente movimiento de las personas lleva a que las sociedades se transformen en multiculturales, donde se requiere no solo el dominio de la lengua del otro, sino también desarrollar una comprensión cultural del otro. Por lo tanto, es necesario buscar o abrirse a nuevas estrategias para facilitar la comprensión y el entendimiento entre quienes conforman la sociedad, independiente del idioma en el que se comunican y los rasgos culturales que los identifican. Es claro que el desconocimiento entre las personas que conforman una sociedad puede conducir a una comprensión errada del comportamiento del otro, provocando malentendidos y conflictos durante el acto comunicativo; esto puede surgir debido a la poca comprensión del contexto en el que surge la interacción, como también al realizar interpretaciones según los referentes culturales propios, que difieren a los pertenecientes de aquellos cuya lengua es la meta.

En ocasiones, los errores culturales provocan mayor complejidad durante el acto comunicativo, que los errores en el uso de alguno de los elementos lingüísticos; es más, cuando se cometen errores que implican estructura sintáctica y de pronunciación, los asociamos al desconocimiento de la lengua y, en general, se comprende esa deficiencia. Sin embargo, cuando existen errores relativos a la forma de comportarse, de mirar, de mover las manos, se piensa que se debe a problemas con el hablante, que es este quien tiene dificultades para relacionarse con una persona que no es parte de su contexto cultural.

Por lo tanto, es crucial, como base del aprendizaje de una lengua, que quienes participen del aula conozcan anticipadamente el contexto de aquellos con quienes se compartirían saberes.

Así mismo, los participantes deben constituirse en gestores de los aprendizajes a ser desarrollados, según como lo establecen Robalino, Leyton, Basoalto, Beyer y Soto (2005), quienes señalan que son los aprendices quienes deben desempeñar un rol fundamental en la construcción de sus aprendizajes; y para que se desarrollen los aprendizajes de manera significativa, el programa debe ser una construcción social. Esto implica situar la enseñanza en un contexto de necesidades e intereses específicos de cada comunidad involucrada.

Los resultados muestran que mediante el programa de adquisición de la lengua criollo haitiana, llevado a cabo en Talca, Chile, se logran la competencia comunicativa a nivel A1 y la competencia comunicativa intercultural, con base en la interrelación de los elementos lingüísticos y culturales propios de los participantes. De igual modo, los aprendices valoran la propuesta educativa, donde no existe en el ser ni en el hacer una concepción de una figura jerárquica única durante el proceso educativo, sino que se evidencia una coexistencia de las diversidades entre los participantes, durante cada encuentro. Lo anterior se relaciona con lo que señala Freire (2009) acerca del acto educativo, donde el diálogo y el ámbito social se establecen entre iguales, donde el que tiene el rol de conductor no ejerce un papel autoritario ni directivo, sino que es generador de las instancias de (co)construcción del conocimiento y traspaso de saberes lingüísticos y culturales. Al final del programa, cada participante es una individualidad reconocible y valorada por sus conocimientos y saberes.

\section{Referencias}

Antón, M. (2010). Segunda lengua, evaluación dinámica e inclusión educativa en Estados Unidos. Revista de Educación Inclusiva, 3(1), 51-66.

Areizaga, E. (2001). Cultura para la formación de la competencia comunicativa intercultural: el enfoque formativo. Revista de Psicodidáctica, (11-12), 157-170.

Arroyo, M. J. (2012). Las aulas de inmersión lingüística para alumnado inmigrante en el marco de la escuela inclusiva: algunas propuestas de mejora. Tendencias Pedagógicas, (19), 25-42.

Bisquerra, R. (Coord.) (2009). Metodología de la investigación educativa (2. ${ }^{\mathrm{a}}$ ed.). Madrid: La Muralla S. A.

Bohr, N. (1958). Atomic Physics and Human Knowledge. New York: Wiley.

Byram, M., Gribkova, B., y Starkey, H. (2002). Developing the Intercultural Dimension in Language Teaching: 
A Practical Introduction for Teachers. Strasbourg: Council of Europe.

Chen, G-M., y Starosta, W. J. (1998). Foundations of Intercultural Communication. Boston: Allyn and Bacon.

Chile, Instituto Nacional de Estadísticas (INE). (2018). Sintesis de resultados Censo 2017. Recuperado de http://www.censo2017.cl/descargas/home/sintesis-de-resultados-censo2017.pdf

Chiswick, B. y Miller, P. (2001). A model of destination-language acquisition: Application to male immigrants in Canada. Demography, 38(3), 391-409. https:// doi.org/10.1353/dem.2001.0025

Cummins, J. (1979). Linguistic interdependence and the educational development of bilingual children. Review of Educational Research, 49(2), 222-251. https://doi.org/10.2307/1169960

Cummins, J. (1981). The role of primary language development in promoting educational success for language minority students. In California State Department of Education (Ed.), Schooling and Language Minority Students: A Theoretical Framework (pp. 3-49). Los Angeles: Center California State University.

Cunliffe, A. L. (2010). Crafting qualitative research: Morgan and Smircich 30 Years On. Organizational Research Methods, 14(4), 647-673. https://doi. org/10.1177/1094428110373658

Díaz, F. (2003). Cognición situada y estrategias para el aprendizaje significativo. Revista Electrónica de Investigación Educativa, 5(2), 1-13. Recuperada de https://redie.uabc.mx/redie/article/view/85/1396

Ferrada, D., y Flecha, R. (2008). El modelo dialógico de la pedagogía: un aporte desde las experiencias de comunidades de aprendizaje. Estudios Pedagógicos, 34(1), 41-61. http://dx.doi.org/10.4067/ S0718-07052008000100003

Freire, P. (2009). La educación como práctica de la libertad. Madrid, España: Siglo XXI editores.

Hernández, R., Fernández, C., y Baptista, M. (2014). Metodología de la investigación (6. ${ }^{\mathrm{a}}$ ed.). México: McGraw Hill.

Huguet, Á. (2008). Interdependencia lingüística y práctica educativa. Un estudio con escolares de origen inmigrante. Infancia y Aprendizaje, 31(3), 283-301. https://doi.org/10.1174/021037008785702901

Huguet, Á. (2014). Latin American students and language learning in Catalonia: What does the linguistic interdependence hypothesis show us? Spanish Journal of Psychology, 17, 1-13. https://doi.org/10.1017/ sjp. 2014.11
IBM SPSS Statistics (2009). Recursos para sPss statistics. Recuperado de https://www.ibm.com/cl-es/products/ spss-statistics/resources

Instituto Cervantes (2002). Marco común europeo de referencia para las lenguas: aprendizaje, enseñanza, evaluación. Madrid: Instituto Cervantes. Recuperado de https:// cvc.cervantes.es/ensenanza/biblioteca_ele/marco/ cvc_mer.pdf

Lave, J., y Wenger, E. (1991). Situated Learning: Legitimate Peripheral Participation. Cambridge: Cambridge University Press.

León, G. (2014). Aproximaciones a la mediación pedagógica. Revista Calidad en la Educación Superior, 5(1), 136-155. Recuperado de http://investiga.uned. ac.cr/revistas/index.php/revistacalidad/article/ view/348/249

Littlewood, W., y Yu, B. (2011). First language and target language in the foreign language classroom. Language Teaching, 44(1), 64-77. http://dx.doi. org/10.1017/S0261444809990310

Maalouf, A. (Coord.). (2008). Un reto provechoso. Cómo la multiplicidad de lenguas podría contribuir a la consolidación de Europa. Bruselas: Comisión Europea.

Moreno, F. (2013). Lingüística y migraciones hispánicas. Lengua y migración, 5(2), 67-89.

Navarrete, B. (2015). Factores explicativos de una oleada migratoria. El caso de Haití. Revista de Ciencias Sociales, 21(1), 97-107. Recuperado de https://www. redalyc.org/pdf/280/28037734009.pdf

Oller, J., y Vila, I. (2008). El conocimiento de catalán y castellano del alumnado de origen extranjero, tiempo de estancia en Cataluña y lengua inicial al finalizar la enseñanza Primaria. Segundas Lenguas e Inmigración en Red, 1, 11-25. Recuperado de https://dialnet.unirioja.es/descarga/articulo/2797905.pdf.

Paricio, M. S. (2014). Competencia intercultural en la enseñanza de lenguas extranjeras. Porta Linguarim, 21, 215-226. Recuperado de https://www.ugr. es/ portalin/articulos/PL_numero21/14\%20\%20 Silvina.pdf

Policía de Investigaciones (PDI) (2018, 1 de abr.). Ingreso de extranjeros sigue aumentando, en medio de intenso debate migratorio. El Mercurio, p. D4. Recuperado de https://www.google.com/search?q=Ingreso+de+extr anjeros+sigue+aumentando, + en + medio + de + intenso +debate+migratorio\&rlz=1C1CHBF_esCL873CL8 73\&sxsrf=ACYBGNTzwQJCAdCNvLkibcKjOGfe QM5-aQ:1575899046958\&source=lnms\&tbm=isc h\&sa=X\&ved=2ahUKEwi3hpW32ajmAhW8K7kG 
HdTzAd4Q AUoAnoECAwQBA\&biw=1366\&bi $\mathrm{h}=657$ \#imgrc $=\mathrm{uJouPtdiXIAcRM}$

Quintriqueo Millán, S., y McGinity Travers, M. (2009). Implicancias de un modelo curricular monocultural en la construcción de la identidad sociocultural de alumnos/as mapuches de la IX Región de La Araucanía, Chile. Estudios Pedagógicos, 35(2), 173-188. http:// dx.doi.org/10.4067/S0718-07052009000200010

QSR International (2014). NVivo 10 for Windows Primeros Pasos. Recuperado de http://download. qsrinternational.com/Document/NVivo10/NVivo10-Getting-Started-Guide-Spanish.pdf

Ramos, F. (2006). Los programas bilingües inglés-español en Estados Unidos y en España: dos innovaciones en la enseñanza de idiomas. En 400 años de Don Quijote: pasado y perspectivas de futuro. Actas del XL Congreso Internacional de la Asociación Europea de Profesores de Español (pp. 333-342). Universidad de Valladolid, 25 al 30 de julio de 2005.

Rey von Allmen, M. (2010). The intercultural development and its development through cooperation with the Council of Europe. En C. A. Grant y A. Portera (Eds.), Intercultural and Multicultural Education. Enhancing Global Interconnectedness (pp. 33-48). Nueva York: Routledge.

Robalino, M., Leyton, M., Basoalto, V., Beyer, H., y Soto, V. (2005). Distintas miradas sobre el currículo y el rol docente. Revista Docencia, (25), 49-57.

Rodríguez Gómez, G., Gil Flores, J. y García Jiménez, E. (1999). Metodología de la investigación cualitativa. Málaga: Algibe.

Rojas Pedemonte, N., Silva, C., Amode, N., Vásquez, J. y Orrego, C. (2016). Boletín informativo $n^{\circ} 1$. Migración haitiana en Chile. Departamento de Extranjería y Migración. Recuperado de http://www.extranjeria.gob.cl/media/2016/09/boletin-1.pdf
Rojas Pedemonte, N., y Koechlin, J. (2017). Migración haitiana hacia el sur andino. Perú: Litho \& Arte S.A.C.

Sancho, M. (2013). La integración sociolingüística de la inmigración hispana en España: lengua, percepción e identidad social. Lengua y migración, 5(2), 91-110.

Santibáñez Gaete, G. P., y Maza Espinoza, C. M. (2015). Ser niño haitiano (que no habla español) en una escuela chilena (tesis de pregrado). Facultad de Artes, Escuela de Teatro Universidad Academia de Humanismo Cristiano, Santiago, Chile.

Serra, J. M., y Vila, I. (2005). Lenguas, escuela e inmigración en Catalunya. En D. Lasagabaster y J. M. Sierra (Eds.), Multilingüismo, competencia lingüistica $y$ nuevas tecnologías (pp. 75-89). Barcelona: Horsori.

Sumonte, V., Sanhueza, C., Friz, M., y Morales, K. (2018). Migración no hispanoparlante en Chile: tendiendo puentes lingüísticos e interculturales. Diálogo Andino, 57, 57-67. http://dx.doi.org/10.4067/ S0719-26812018000300061

Toledo, G. (2016). Propuesta didáctica para la enseñanza de español como segunda lengua a inmigrantes haitianos en Chile. Lengua y migración, 8(1), 81-103.

Tristán, A. (2008). Modificación al modelo de Lawshe para el dictamen cuantitativo de la validez de contenido de un instrumento objetivo. Avances en Medición, 6(1), 37-48.

Universidad de Chile (2016). La enseñanza del español desde la U. de Chile para el mundo. Recuperado de http:// www.uchile.cl/noticias/122197/la-ensenanza-delespanol-desde-la-u-de-chile-para-el-mundo

Vigotsky, L. (1993). Obras escogidas (Tomo 2). Madrid: Aprendizaje Visor.

Vilà, R. (2009). La competencia comunicativa intercultural en alumnos de Enseñanza Secundaria de Catalunya. Segundas Lenguas e Inmigración en Red, 3, 88-108. Recuperado de https://dialnet.unirioja.es/descarga/articulo/3186901.pdf

How to reference this article: Sumonte-Rojas, Valeria (2020). Desarrollo de la competencia comunicativa intercultural en un programa de adquisición de la lengua criollo haitiana en Chile. Íkala, Revista de Lenguaje y Cultura, 25(1), 155-169. http://doi.org/10.17533/udea.ikala.v25n01a09 